\title{
Generalization Ability of Deep Learning Algorithms Trained using SEM Data for Objects Classification
}

\author{
Yasmina Zaky, Nicolas Fortino, Benoit Miramond, Member, IEEE, and Jean-Yves Dauvignac, Member, IEEE.
}

\begin{abstract}
This paper proposes a workflow to efficiently determine the material of spherical objects and the location of the receiving antenna relative to their position in bi-static measurements using supervised learning techniques. From a single observation, we compare classification performances resulting from the application of several classifiers on different data types: the Ultra-Wide Band scattered field in time and frequency domains and pre-processed data from the Singularity Expansion Method (SEM). Indeed, the resonances extracted using the SEM are aspect independent and therefore, are used to discriminate the objects. As for the residues, they depend upon the aspect angle and can hence be exploited to determine the observation angle. We construct 3 datasets to assess which one yields the highest accuracy while using the simplest and fastest classifiers. Hence, $\mathbf{8 0 \%}$ of each dataset is used for training and the remaining $20 \%$ are used for testing. In a further step, we test with sphere sizes and data with several noisy levels that were not in the training datasets. Although SEM is noise sensitive, associating a robust feature extraction technique (Vector Fitting) with a simple but reliable classifier is promising, particularly when generalizing to data not included in the training set.
\end{abstract}

Index Terms-Singularity Expansion Method, Vector Fitting, Decision Trees, Support Vector Machines, neural networks, natural resonances, frequency response, radar target recognition, radar target classification.

\section{INTRODUCTION}

$\mathbf{R}$ ECOGNITION and classification of targets is a challenging task in the radar signal processing area. In recent years, Machine Learning (ML) and Deep Learning (DL) algorithms have become increasingly popular where extensive studies have been conducted by researchers for classification purposes. Several algorithms are found in literature, that vary from simple models, like binary or multi-class Support Vector Machines (SVM) or Decision Trees (DT) [1]-[3], to more complex models like Artificial Neural Networks (ANNs) [4].

In fact, multiple data types are used for classification. Radar Cross Section (RCS) responses can be directly used as raw data, as in [5], [6], where authors used them to classify objects using Convolutional Neural Networks (CNNs) and SVM respectively. Moreover, Micro-Doppler measurements are used for the detection of humans and the classification of moving targets or human activities [7], [8]. Radar imaging techniques can also be applied to generate pre-processed data where the Synthetic Aperture Radar (SAR) images are deployed for classification [9], [10]. However, this technique requires multiple observation angles to form an image, making it unsuitable for our application as we seek to classify targets through a single observation angle. Additionally, although the previous techniques can achieve high accuracy results in target classification, they still require the use of large classifiers.
There exists another approach for classification based on the natural resonant frequencies of an object, extracted from its Ultra-Wide Band (UWB) scattered electromagnetic (EM) field response, which is the Singularity Expansion Method (SEM) [11]. This method is used to compute the characteristic poles of targets from the late time response of their UWB scattered field and thus allowing to identify them when illuminated by a plane wave. These poles are intrinsic to the studied object and are independent of the incident and aspect angles, making the SEM a very interesting method in an operational context when the target position is not completely controlled. In fact, the SEM is widely used for identification where it has been applied to study the extracted natural frequencies from Perfect Electric Conductor (PEC) objects [12], [13] or dielectric objects [14]-[16]. It is also used to measure the maturity of fruits as in [17], [18]. Conversely, the SEM has been hardly used for classification purposes: authors in [19] proposed the use of natural resonances in a bi-class classification task using an ANN classifier. They show that this approach renders the classification more robust than the direct use of time responses because the resonances are aspect independent. However, there are limitations where the resonances are very sensitive to noise. Then, in [20], authors proposed to apply the Principal Component Analysis (PCA) to noisy signals and then extract the resonances with the SEM technique, using Matrix Pencil method in time domain, to reduce the effects of noise. They validated this procedure on the classification of four different sizes (four classes) of different objects separately, making the classification task not very complex. In fact, the value of the first natural pole is inversely proportional to the size of the object, hence, it is sufficient to classify between homothetic objects. In addition, the learning process in both references was carried out at different Signal-to-Noise Ratios (SNRs) to improve the robustness of the classifiers to noise.

Our first objective is to address the multi-class classification of spherical objects using supervised learning techniques to discriminate spheres of different materials. For this purpose, we construct datasets using only noiseless data: two of them are based on UWB scattered field (SF) (in time and frequency domain) computed using Mie series and a third one based on SEM data extracted using Vector Fitting (VF) algorithm. Particular consideration will be given to the constitution of the SEM dataset to obtain compact, informative, and generalizable data. The optimal association of the SEM dataset with different classification algorithms and the robustness to noise will also be discussed. In addition to the extracted natural poles with the SEM, other parameters are extracted which are the residues associated to each pole. Those residues are dependent upon 
the aspect angle and have therefore not been exploited. In fact, as the residues vary with the observation angle, they can be considered for location detection of the observer when doing measurements in a bi-static manner. For that, our second objective is to determine the observation angle of the receiving antenna by dividing the sphere into multiple angular sectors.

The paper is organized as follows: In section II, we present a brief description of each classifier used in this work. Section III deals with the generation of UWB scattered fields for multiple sphere types and the pre-processing step to extract the singularities using VF. Then, in section IV, we start by classifying the sphere's material where we construct the datasets from SF and SEM data. We also show how the algorithms generalize to other sphere dimensions and noisy data not included in the training datasets. Following, in section $\mathrm{V}$, we present the classification of observation angle of the simulated spheres using raw SF data and the residues. To our knowledge, it is the first time that residues have been used to determine the position of the observer relative to an object. Finally, we discuss and conclude the work in sections VI and VII respectively.

\section{SUPERVISED ML ALGORITHMS}

In this section, we briefly explain each one of the classifiers used within this work. The SVM and DT algorithms are implemented with built-in functions in Python using scikit-learn library [21]. The ANNs are implemented using the Keras-Tensorflow library tool in Python.

\section{A. Multi-class SVM}

The SVM is originally a binary classifier that consists in finding the optimal hyperplane to separate different data classes. Under the assumption that the data are linearly separable, this plane is found by solving the problem in [1]. If they are not linearly separable, SVMs employ the kernel method to map the data onto a higher dimensional space where they become linearly separable [1]. In order to apply the SVM in a multi-class configuration, we use the one vs one (ovo) approach [2]. This concept is based on constructing $k(k-1) / 2$ classifiers and then treating the problem as a binary one.

The hyperparameters of the SVM are $C$ and $\gamma . C$ is a regulation parameter, which adjusts the width of the margin to minimize the training error. $\gamma$ is the data variance. Here, we apply the five-fold cross-validation using 'gridsearch' function in order to find the optimal kernel function, the optimal values of $C$ and $\gamma$ and to avoid over-fitting.

\section{B. Decision Tree}

DT methods build a decision model based on the actual values of attributes in the data. The dataset is recursively divided into smaller homogeneous subsets, resulting in a tree. They use various techniques to split the data like Gini, information gain or entropy [3]. The minimum amount of samples required to split a decision node is two.

\section{Artificial Neural Network}

1) Multi-Layer Perceptron: One of the well-known ANN models is the Multi-Layer Perceptron (MLP). It comprises an input layer with a number of neurons equals to the number of input data which simply pass information to the next layer; one or several hidden layers composed of various amount of neurons; and an output layer with a number of neurons equal to the number of classes. The neurons of the hidden layers and the output layer are called perceptrons. A perceptron is a neuron that is connected to the output of the previous layer and whose output is connected to the neurons of the next layer. It uses a non-linear activation function that is applied to a sum of products of the inputs related to their weights [4].

2) Convolutional Neural Network: CNNs are composed of multiple layers of different types. Usually, the most common layers are convolution, pooling, and fully-connected layers. The Convolution layer is a filter layer, of specific length and width, that moves along the input data. It is composed of kernels or filters that are applied to extract specific features from the input vector [4]. The pooling layer is a sub-sampling layer used to reduce the size of the features computed using the convolution layer. Here, we use the maximum pooling layer that outputs the maximum input value. Fully-connected layers are used to process the output features of the last convolution or pooling layer. They are composed of neurons connected to all previous layer's neurons.

\section{SCATTERED FiELD DATA GENERATION AND PRE-PROCESSING}

The UWB SF from 5 different classes of spheres is computed in the far-field region from 0.01 to $5 \mathrm{GHz}$, for both mono and bi-static configurations, using Mie series algorithm implemented in Matlab [22]. The UWB scattered fields were measured at a distance of $1 \mathrm{~m}$, considering that it is possible to estimate and compensate the propagation channel in the far field at a distance $r$.

$$
\underline{\vec{E}}(\vec{r}, \theta, \phi)=\frac{e^{-j \vec{k} \vec{r}}}{r} \cdot \overrightarrow{E^{0}}(\theta, \phi)
$$

The SF is recovered for both $\theta$ and $\phi$ polarizations by decomposing $\overrightarrow{E^{0}}(\theta, \phi)$ into $\overrightarrow{E_{\theta}}$ and $\overrightarrow{E_{\phi}}$. The 5 classes of spheres are: PEC sphere, sphere with a relative permittivity $\epsilon_{r}=4$ and conductivity $\sigma=0.5$, three lossless dielectric spheres with $\epsilon_{r}$ equal to 2, 4 and 9 . They are enumerated from 0 to 4 respectively. All spheres are illuminated by an $x$-polarized incident plane wave propagating along $z$ axis. The SF is recovered for multiple observation angles where $\theta$ varies from $0^{\circ}$ to $180^{\circ}$ with $5^{\circ}$ step and $\phi$ from $-180^{\circ}$ to $180^{\circ}$ with $10^{\circ}$ step. $\theta$ and $\phi$ are defined from the standard 3D cartesian coordinate system. Fig. 1 shows the amplitude of the SF in the back-scattering direction $\left(\theta=180^{\circ}\right)$ for the 5 simulated sphere classes of $10 \mathrm{~cm}$ diameter.

The pre-processing of the SF is done using the SEM technique applied on each simulated object. The SEM data are extracted by applying VF in frequency domain [23]. Indeed, it was shown in [24] that VF is more robust to noise and more accurate than other SEM techniques. It is a widely used 


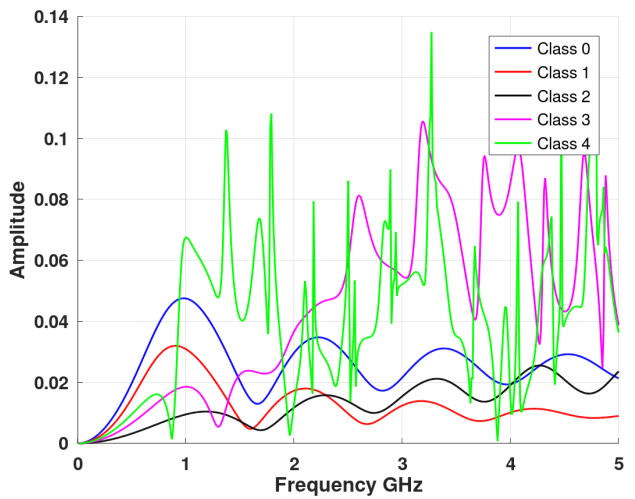

Fig. 1: Amplitude of the frequency response in the back-scattering direction for the 5 simulated sphere classes of $10 \mathrm{~cm}$ diameter

method for fitting a measured or simulated frequency domain response $H(s)$ using the following rational function:

$$
H(s)=\sum_{m=1}^{M} \frac{r_{m}}{s-a_{m}}+d+s * e
$$

with $M$ being the model order, $a_{m}$ the resonant poles and $r_{m}$ the residues. $d$ and $e$ are optional real constants (fixed to 0 in our case) [23].

The first step is to identify the poles by assuming a set of starting complex poles that are uniformly distributed over the frequency band of interest. Then, through successive iterations, the algorithm relocates them towards the actual poles of resonance [25], [26]. If the data are noise free, one or two iterations are enough to get accurate poles under the condition that the number of starting poles is greater than $M$. In presence of noise, further iterations are needed for convergence. Once the poles have been determined, the residues can be computed by solving the corresponding Least Square (LS) problem.

We also compute the quality factor (Q-factor) of each pole; as in [27]; to replace the damping factor. This parameter is an important representation of the object as it is independent of its size which will help in generalizing the classification to spheres of different sizes. It is computed as follows:

$$
Q_{m}=-\frac{2 * \pi * f_{m}}{2 * \alpha_{m}}
$$

where $f_{m}$ is the frequency of resonance of the $m_{t h}$ singularity, and $\alpha_{m}$ is its damping factor. From Fig. $2 \mathrm{a}$ and $2 \mathrm{~b}$ we see that all classes of $10 \mathrm{~cm}$ diameter spheres exhibit 5 poles in the frequency range of interest. When the relative permittivity of the dielectric material increases, the sphere becomes a very strong resonating object, hence, it has a very low damping factor and a very high Q-factor. In Fig 2b, we do not show the rest of the Q-factors for both class 3 and 4 for visibility as they are higher than 15 .

To construct the datasets, 13 sphere dimensions are simulated for each of the 5 classes. The dimensions are selected to ensure that each sphere exhibits at least one natural frequency within the previously determined frequency band of interest. Table I shows the simulated sphere diameters where
TABLE I: 13 Simulated sphere diameters $(\mathrm{cm})$ having different \# of resonances $(N)$ for each sphere class

\begin{tabular}{llllll}
\hline \multirow{2}{*}{ Class } & \multicolumn{5}{c}{$\mathbf{N}$} \\
& $\mathbf{1}$ & $\mathbf{2}$ & $\mathbf{3}$ & $\mathbf{4}$ & $\mathbf{N} \geq \mathbf{5}$ \\
\hline 0) $\mathbf{P E C}$ & 3 & 5 & 7 & 8.5 & \\
1) $\epsilon_{r}=\mathbf{4}, \sigma=\mathbf{0 . 5}$ & 3 & 4.5 & 6 & 8 & \\
2) $\epsilon_{r}=\mathbf{2}$ & 4.5 & 6 & 8 & 9 & {$[10: 1: 18]$} \\
3) $\epsilon_{r}=\mathbf{4}$ & 3.5 & 5 & 6 & 7 & \\
4) $\epsilon_{r}=\mathbf{9}$ & 2.5 & 3.5 & 4.3 & 5 & \\
\hline
\end{tabular}

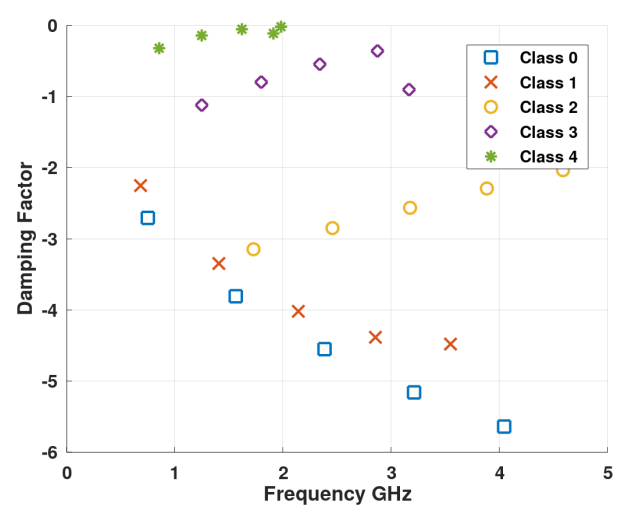

(a)

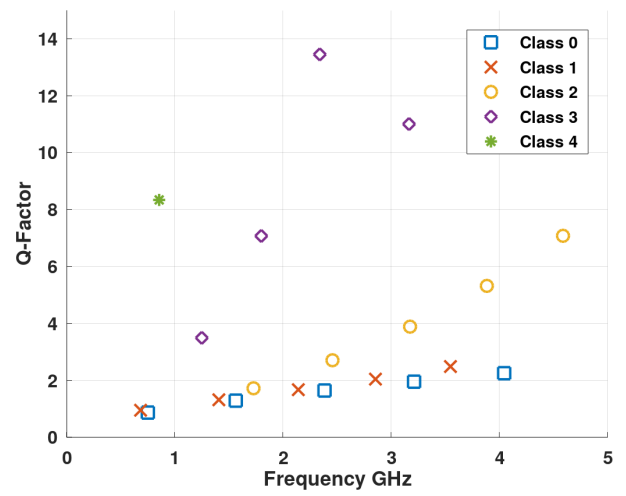

(b)

Fig. 2: (a) The first five natural poles and (b) Q-factors as a function of natural frequency of the 5 spheres of $10 \mathrm{~cm}$ diameter.

$N$ represents the number of resonances in the frequency range. For spheres having $N \geq 5$ resonances in the frequency band, we chose the diameters varying from $10 \mathrm{~cm}$ to $18 \mathrm{~cm}$ with a $1 \mathrm{~cm}$ step, resulting in 9 objects for each class. Those 9 sizes are mostly useful for generalization using raw data but are usually useless for data pre-processed with SEM. For spheres with $N<5$ resonances, we have a different diameter for each sphere class that corresponds to each case of $N$ resonance.

\section{ClassificATION OF SPHERE MATERIAL}

In this part, we describe the construction of three different datasets that will be used to feed the classification algorithms. The first two datasets are directly composed of the SF responses in frequency and time domain respectively. The 3rd dataset is the one integrating data pre-processed by the SEM from these scattered fields. The constitution of these datasets is of primary importance as it directly impacts the performance 
of the classification algorithms. The comparative results using different classifiers fed with these datasets will be presented and discussed in this section.

\section{A. Dataset construction}

We start by creating datasets including the 5 sphere classes simulated in section III. To reduce the size of the datasets, we include data from only three main planes; $\phi=0^{\circ}$ and $\theta$ varies ( $X \circ Z$ plane), $\phi=90^{\circ}$ and $\theta$ varies ( $Y o Z$ plane), $\theta=90^{\circ}$ and $\phi$ varies ( $X \circ Y$ plane). It should be noted that creating datasets with all observation angles yields similar classification results. Thus, for each sphere size we have 111 observation angles (3 planes and 37 angles in $\theta$ or $\phi$ directions), making a total of 7215 samples for each of the three datasets as we have 13 sphere sizes for each of the 5 classes.

1) Scattered Field dataset: We construct a first dataset using the amplitude of the simulated SF in frequency domain. The classifiers used within this work do not support complex numbers, thus, we include only the amplitude response. A preliminary study showed that adding the phase to this dataset was not relevant as it doubled its size without improving its performance. The input vector is, hence, a 1-D signal of 500 frequency points and composed of 2 channels. The first channel represents the $\theta$ component and the second one is the $\phi$ component of the SF.

The second dataset represents the SF in time domain. The transient impulse response is computed using inverse Fourier transform of the complex SF. Here, we include the first $10 \mathrm{~ns}$ of each signal, which constitutes 100 time points, as it starts to decay after. The input vector is also a 1-D signal of 100 points and composed of 2 channels, one for each polarization.

2) SEM dataset: To construct this dataset, we pre-process the complex frequency response using VF as described in section III. The first five natural frequencies of each object and their respective Q-factors and residues are chosen to form this dataset's input vector. For the smaller spheres having less than 5 resonances in the frequency range (see table I), we will need to complement the missing data with zeros to preserve the same input vector's length. Hence, we will have sparse SEM data in this dataset. This original configuration is chosen to improve the generalization performance of the classifiers when limiting factors (limited bandwidth, noise, etc.) impact the higher order poles.

The input vector is, thus, of length 5 and composed of 4 channels: the natural frequencies, the Q-factor and the amplitude of residues of $\theta$ and $\phi$ components respectively. The residues are included even though they are aspect dependent, as they contain additional information about the objects which can be significant when the natural frequencies and Q-factors are almost similar for some objects. We also test more conventional approaches for building the SEM dataset to find the optimal one. Thus, we will have the following three cases:

- case 1: An original dataset as described above (four channels: natural frequencies, Q-factors and their respective residues);
- case 2: Q-factor is replaced by the damping factor (four channels: natural frequencies, damping factors, and their respective residues);

- case 3: we eliminate the residues from case 1, hence, the input vector has only the first two channels (natural frequencies and Q-factors only).

In the rest of the paper, we will use the following abbreviations to refer to the datasets: FD data, TD data, SEM data which are frequency domain, time domain and SEM data respectively. Note that case 1, 2 and 3 only refer to SEM data.

\section{B. Classification Results}

Each dataset is split into $80 \%$ for the training and $20 \%$ for the testing. The remaining $20 \%$ are composed of random observation angles where each class has an equal number of samples. In a further step, we test noisy data where they are affected by a white Gaussian noise with different SNRs. Following, we present results of the generalization ability of the classifiers. We begin with spheres with diameters higher than those included in the training datasets $(>18 \mathrm{~cm})$. Increasing the sphere's diameter means, physically, that the first 5 natural frequencies are shifted down into the frequency band. For that, we simulate new spheres of 19 and $30 \mathrm{~cm}$ diameter for each class. We also simulate smaller spheres with less than 5 resonances and different than those simulated in table I (but always smaller to test the generalization capabilities with natural frequencies being shifted up into the frequency band). Results presented are averaged over 10 runs.

1) Training Phase: During this phase, the parameters of each classifier are tuned to achieve highest accuracy results for training data. First, the SVM parameters are fixed according to the results using 'gridsearch' function. For that we defined, for each dataset, a matrix of $C$ and $\gamma$ values, two kernel functions: Linear and Radial Basis Function (rbf) and ran the 5-fold cross-validation. Results of optimum values are given in table II. Notice that the training parameters are much smaller for SEM dataset where it uses a simple linear function, because the data are easily separable compared to the other datasets.

TABLE II: Parameters of SVM classifier for the 3 datasets

\begin{tabular}{cccc}
\hline \multirow{2}{*}{ Data } & \multicolumn{3}{c}{ Parameters } \\
& Function & C & $\gamma$ \\
\hline FD & rbf & 2500 & 10 \\
TD & rbf & 1500 & 10 \\
SEM & Linear & 5 & - \\
\hline
\end{tabular}

Second, for the DT algorithm, the Gini criterion is used to measure the quality of the split in the tree and decision nodes are randomly chosen to be further split.

Then, when using the MLP classifier, we apply one hidden layer while varying the number of neurons. Note that we have done tests with other topologies by increasing the number of hidden layers and have found that the performances are almost similar. We use the tanh activation function with SEM data and Rectified Linear Unit (ReLU) for SF data. The learning rate is updated using the Adam optimizer [28]. Finally, the CNN is tested. For SF data we adopt the LeNet-5 architecture [29] where we change the filter size to be applied on 1-D 


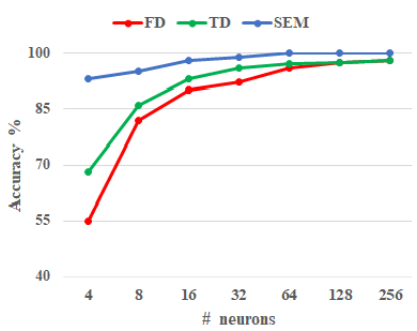

(a)

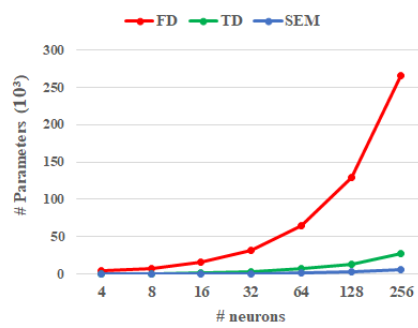

(b)
Fig. 3: (a) Mean accuracy of the 5 spheres; (b) \# Parameters vs. \# neurons in one hidden layer for the 3 datasets.

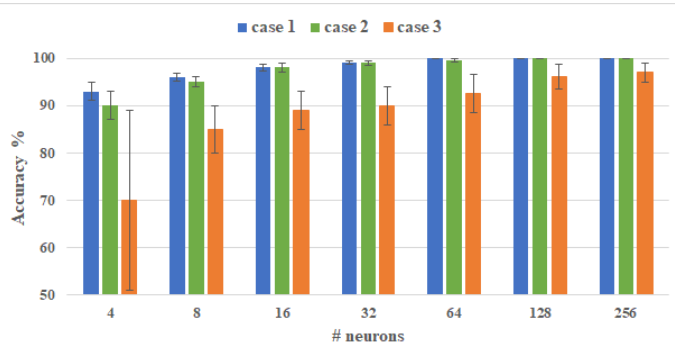

Fig. 4: Test results of 3 cases of SEM dataset construction using MLP classifier while varying \# neurons.

input signals. For SEM data, we apply one convolutional layer with 6 filters, followed by one hidden layer with 32 neurons and ReLU function applied in all layers. This is run over 100 epochs for SEM data and 400 epochs for SF data with a batch size of 32 for both MLP and CNN classifiers. They both have an output layer composed of 5 neurons using the softmax activation function to compute the output probabilities.

2) Test Phase: First, we present the mean accuracy results when testing the $20 \%$ remaining samples of each dataset for various amount of neurons using MLP classifier. In fig.3a, we can see that, for SEM data (case 1), 64 neurons are enough to get $100 \%$ accuracy and for SF datasets, 256 yields the highest accuracy of $98 \%$. The number of parameters (weights) computed in the hidden layer increases with the number of neurons, as shown in fig. 3b where the FD data has the highest parameters as it has the longest input vector. Thus, we can see that the SEM dataset has both the highest accuracy and the lowest parameters computed using the MLP. In addition, we test the 3 cases of SEM dataset construction. Fig. 4 shows that the MLP classifier trained using SEM data of both cases 1 and 2 has a higher accuracy (100\%) for a number of neurons $>$ 32 and is more stable (low variance) than the classifier trained without the residues. In fact, from fig. $2 \mathrm{~b}$, we see that some classes have very close natural frequencies and Q-factor, that is why, when trained with case 3 without residues, it becomes more difficult to separate those classes. We found that DT and SVM classifiers have similar results but with a higher variance when trained without residues. Thus, for the rest we will only work with SEM datasets of case 1 and 2.

The performances of the classifiers are also evaluated with the following metrics: the Sensitivity (Sens) and the Specificity (Spec) of each class. Sens is the probability of classifying a
TABLE III: Accuracy (\%) of the $20 \%$ test sets when using the four classifiers with the three datasets

\begin{tabular}{ccccc}
\hline Data & SVM & DT & MLP & CNN \\
\hline FD & 95 & 94.5 & 97 & 98.3 \\
TD & 97.5 & 96.5 & 97.5 & 98.5 \\
SEM case 1 or 2 & 100 & 100 & 100 & 100 \\
\hline
\end{tabular}

sample as True Positive (TP) and Spec is the probability of classifying as True Negative (TN) and they vary from 0 to 1 . They are computed as follows:

$$
\text { Sens }=\frac{T P}{T P+F N} ; \quad \text { Spec }=\frac{T N}{T N+F P}
$$

FN is False Negative and FP is False Positive.

Table III shows the accuracy performances of the classifiers when applied to the three test datasets. We can see that the SEM data (case 1 or 2) yields the highest recognition rate for all classifiers where the Sens and Spec are of 1. Additionally, when using the SF data, CNN model has a higher recognition rate compared to SVM and DT with $1.2 \%$ and $5.2 \%$ gains respectively where the classifiers trained using TD data have higher accuracy than those trained using FD data. In this case, the Sens and Spec are higher than 0.97 for all classes. Fig.5 shows the normalized confusion matrices with respect to the true label for the three datasets when using the CNN classifier. Thus, we see that the classifiers trained using SEM data holding the residues are more capable in distinguishing spheres having close characteristic poles and provide higher accuracy results while consuming much less computational power than classifiers trained using raw data.

3) Noisy test data: Now, we test the classifiers' ability to handle noisy data. Given that generating a dataset with different SNR levels is challenging, we opted to assess the robustness of our classifiers to noise by evaluating them on noisy data that were not seen in the training phase. We chose the $15 \mathrm{~cm}$ diameter sphere as its noiseless response is already included in the training dataset, which should make the classification task using TD or FD data easier. Several white Gaussian noise levels are added to the SF response of the 5 spheres of $15 \mathrm{~cm}$ diameter. The SNR values are chosen such that $65 \mathrm{~dB}$ is one of the highest values that can be obtained in the anechoic chamber, whilst $10 \mathrm{~dB}$ is the lowest. As in the case without noise, VF is applied to extract the poles and residues from the noisy signals. In [24], authors show that the noise highly affects the damping factor values more than the natural frequencies. Consequently, the Q-factor will also be affected by noise as it is computed using the damping factor. Note that when using VF with very noisy signals, a minimum of 10 iterations are needed for good convergence of poles and the model order $M$ should be carefully selected where it should not be too high to avoid numerous poles related to noise that affect the convergence of actual resonance poles.

Accuracy results of the classifiers are shown in fig. 6. For SNR values higher than or equal to $20 \mathrm{~dB}$, both ANN classifiers trained using SEM data (case 1 or 2) achieve high accuracy results. The Sens and Spec are higher than 0.99 for all classes. For 10dB SNR, results start to decrease but are still high and similar to those obtained in [20] for the PEC sphere. However, 


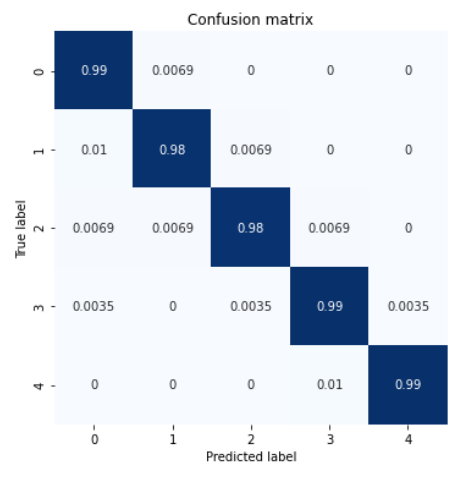

(a)

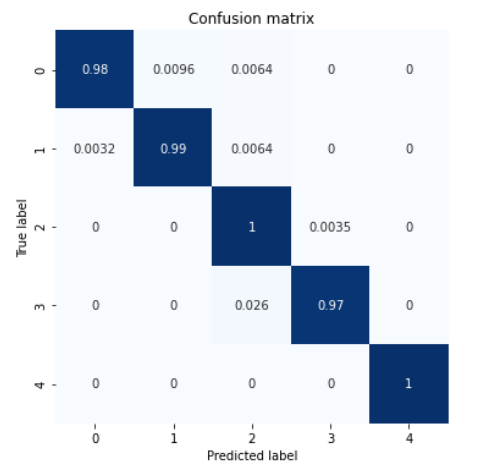

(b)

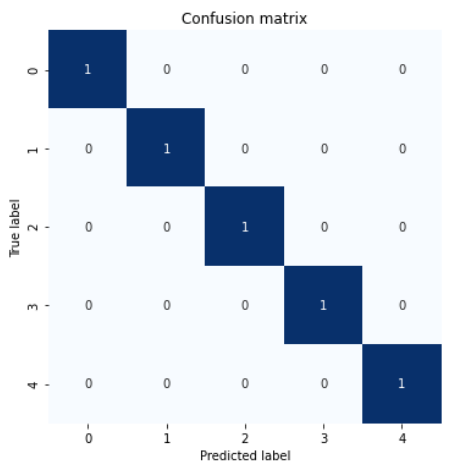

(c)

Fig. 5: Normalized confusion matrix using CNN classifier when testing 20\% remaining samples of : (a) Frequency data ; (b) Time data ; (c) SEM data. The classes are enumerated for each sphere as: (0) PEC; (1) $\epsilon_{r}=4, \sigma=0.5 ;(2) \epsilon_{r}=2 ;(3) \epsilon_{r}=4 ;(4)$ $\epsilon_{r}=9$

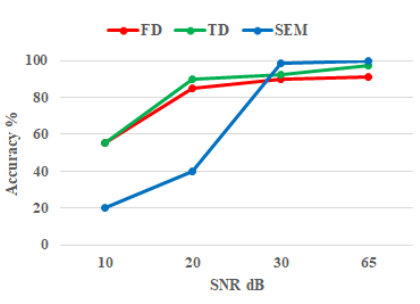

(a) SVM

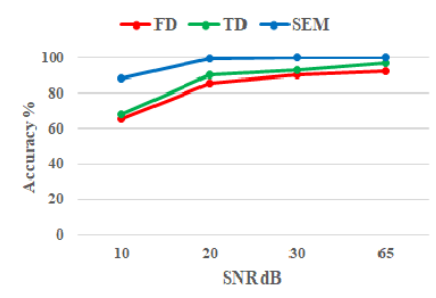

(c) MLP

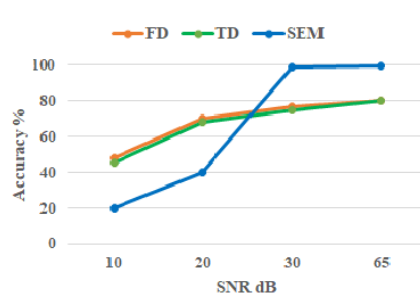

(b) DT

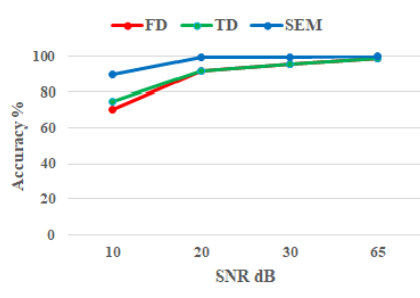

(d) $\mathrm{CNN}$

Fig. 6: Accuracy (\%) of noisy data when testing several classifiers using the 3 datasets

SVM and DT are unable to determine the classes accurately for the $20 \mathrm{~dB}$ and $10 \mathrm{~dB}$ SNR where they have $40 \%$ and $20 \%$ accuracy respectively as they miss-classify all the samples of some classes as in fig 7. Those results can be explained by the fact that, on one hand, the SVM and DT classifiers are basing their decisions only on the Q-factor as it is constant for all sphere dimensions and in the presence of noise it is highly perturbed which in turn perturbs the classification. On the other hand, ANNs are able to classify the noisy SEM data as they are capable of taking advantage of the extra information provided by the original structure of the SEM dataset (sparse data, Q-factor and residues) proposed in this work.

For FD and TD data, we can note that the CNN performs better than the other classifiers and has good performances for high SNR values. However, results start to degrade for SNRs lower than $20 \mathrm{~dB}$ as seen in fig. 6. From table IV, we see that for $10 \mathrm{~dB}$ SNR the Sens and Spec deteriorate for some classes. Hence, we can note that the association of VF with an original input vector structure and ANN classifiers compensates for the

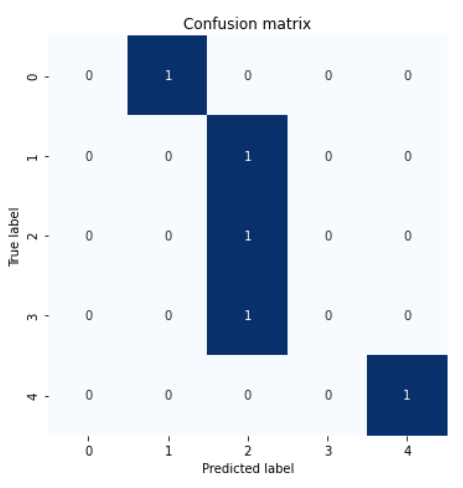

Fig. 7: Normalized confusion matrix when classifying SEM noisy data of $20 \mathrm{~dB}$ SNR with the SVM classifier

TABLE IV: Sens and Spec $(\%)$ for SNR = 10dB using CNN with the 3 datasets

\begin{tabular}{ccccccc}
\hline \multirow{2}{*}{ Class } & & Sens & & \multicolumn{3}{c}{ Spec } \\
& FD & TD & SEM & FD & TD & SEM \\
\hline $\mathbf{0}$ & 32 & 68 & 85 & 97 & 96 & 96 \\
$\mathbf{1}$ & 77 & 72 & 84 & 95 & 93 & 97 \\
$\mathbf{2}$ & 41 & 76 & 93 & 97 & 95 & 99 \\
$\mathbf{3}$ & 90 & 82 & 98 & 72 & 88 & 97 \\
$\mathbf{4}$ & 95 & 77 & 99 & 84 & 90 & 99 \\
\hline
\end{tabular}

noise sensitivity of SEM methods which then outperforms the results obtained using raw data, even at low SNR levels.

4) Generalization using different sphere sizes: We test the generalization ability of all classifiers on larger and smaller spheres. First, for larger spheres, the accuracy results shown in fig. 8 indicate that all classifiers trained using SEM data of case 1 perform an accuracy of $100 \%$ for all classes and for both $19 \mathrm{~cm}$ and $30 \mathrm{~cm}$ diameters. The Sens and Spec are of 1 . These results are due to the Q-factor, associated to each natural frequency which is constant independently of the sphere's size. On the contrary, the SEM data of case 2 did not achieve accuracy results as high as case 1 which shows that replacing the damping factor by the Q-factor is important to be able to properly generalize the classification to sphere sizes larger than those included in the training dataset.

Comparing SEM data (case 1) with TD and FD data, we 


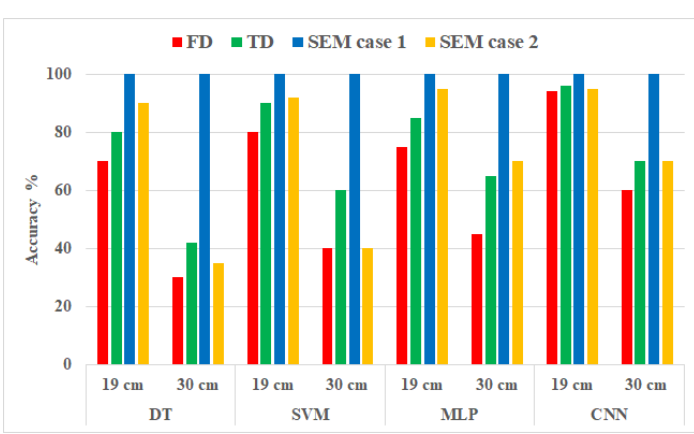

Fig. 8: Accuracy (\%) of 19 and $30 \mathrm{~cm}$ diameter spheres using all classifiers with multiple datasets

TABLE V: Sens and Spec (\%) of $30 \mathrm{~cm}$ diameter sphere using CNN with the 3 data types

\begin{tabular}{ccccccc}
\hline \multirow{2}{*}{ Class } & \multicolumn{3}{c}{ Sens } & \multicolumn{3}{c}{ Spec } \\
& FD & TD & SEM & FD & TD & SEM \\
\hline $\mathbf{0}$ & 38 & 60 & 100 & 88 & 87 & 100 \\
$\mathbf{1}$ & 32 & 67 & 100 & 77 & 85 & 100 \\
$\mathbf{2}$ & 46 & 35 & 100 & 58 & 55 & 100 \\
$\mathbf{3}$ & 43 & 72 & 100 & 58 & 62 & 100 \\
$\mathbf{4}$ & 80 & 79 & 100 & 75 & 78 & 100 \\
\hline
\end{tabular}

find an overall gain of nearly $4 \%$ and $6 \%$ for SEM data respectively with CNN classifier when testing the $19 \mathrm{~cm}$ sphere diameter. Moreover, for larger spheres $(30 \mathrm{~cm}$ diameter $)$, results deteriorate for classifiers trained using raw data, as seen in table V. This shows that classifiers trained using SEM data can be, efficiently, generalized to larger spheres having at least 5 resonances in the frequency range, whereas classifiers trained using SF datasets are unable to classify larger spheres whose sizes are not included in the training dataset.

Second, we test smaller sphere dimensions not included in the training dataset. Table VI shows the accuracy results, using CNN, for data with only $N<5$ resonances in the frequency range as presented in section III (the other classifiers have almost 20\% less accuracy for SF data and same accuracy for SEM data). By using CNN, the sparse SEM data have $0 \%$ error for different $\mathrm{N}$ resonances except for $\mathrm{N}=1$ where it miss-classifies some samples from class 0 as seen in table VII. However, for the SF datasets, when the dimension decreases (i.e. smaller $N$ ), the accuracy decreases drastically where the Sens of some classes are lower than 0.5 for $\mathrm{N}=1$. This shows that the pre-processing of the SF using the SEM method and the computation of the Q-factor to replace the damping factor, is an important step in a classification process in order to distinguish spheres that have different diameters than those included in the training dataset.

Finally, results presented earlier indicate that combining the

TABLE VI: Accuracy (\%) for different number of resonances (N) using $\mathrm{CNN}$ with the 3 datasets

\begin{tabular}{ccccc}
\hline \multirow{2}{*}{ Classifier } & \multirow{2}{*}{ N } & \multicolumn{3}{c}{ Data } \\
& & FD & TD & SEM \\
\hline \multirow{4}{*}{ CNN } & 1 & 42 & 60 & 98.5 \\
& 2 & 56 & 78 & 100 \\
& 3 & 86 & 91 & 100 \\
& 4 & 92 & 95 & 100 \\
\hline
\end{tabular}

TABLE VII: Sens and Spec (\%) for N=1 resonance using CNN

\begin{tabular}{ccccccc}
\hline \multirow{2}{*}{ Class } & \multicolumn{3}{c}{ Sens } & \multicolumn{3}{c}{ Spec } \\
& FD & TD & SEM & FD & TD & SEM \\
\hline $\mathbf{0}$ & 42 & 48 & 93 & 90 & 95 & 100 \\
$\mathbf{1}$ & 68 & 77 & 100 & 77 & 90 & 98 \\
$\mathbf{2}$ & 59 & 68 & 100 & 78 & 84 & 100 \\
$\mathbf{3}$ & 44 & 90 & 100 & 82 & 74 & 100 \\
$\mathbf{4}$ & 21 & 32 & 100 & 90 & 95 & 100 \\
\hline
\end{tabular}

SEM data with MLP or CNN classifier is very robust as it allows to classify spheres with a diameter different than those included in the training phase with a rate higher than $98 \%$ even when there is only one resonance in the frequency band. In addition, it still provides good results in the presence of noise where it outperforms raw SF datasets even at low SNRs. Although the SEM is known for its sensitivity to noise, these performances can be explained by a combination of three major elements. Firstly, the use of VF which is an efficient SEM method chosen for its robustness to noise [24]. Secondly, the sparsity of the proposed original input vector integrating not only the natural frequencies but also their respective residues and Q-factors. Thirdly, the use of powerful ANN classification algorithms which take advantage of this informative dataset and make the difference at low SNR. This association allows to obtain better classification results without adding noisy data in the training step. Moreover, the proposed compact input vector ensures a considerably reduced computational cost than methods using raw SF data.

\section{Classification of ObSERVATION ANGLE}

Now that we have determined the sphere's material, the second stage is to determine the observation angle (i.e. the angle defined by the receiving antenna relative to the transmitting antenna in a coordinate system for which the sphere is the center). For this purpose, before creating the datasets, we split the sphere into 8 angular sectors, where each sector contains various observation angles in $\theta$ and $\phi$ directions, as seen in fig. 9. We only treat one quarter of the sphere as the radiation pattern of the scattered field by the sphere has two symmetry planes. Each angular sector includes the following observation angles:

- sector 0: $0 \leq \phi \leq 90 ; 0 \leq \theta \leq 30$;

- sector 1: $0 \leq \phi \leq 40 ; 35 \leq \theta \leq 65$;

- sector 2: $0 \leq \phi \leq 40 ; 70 \leq \theta \leq 110$;

- sector 3: $0 \leq \phi \leq 40 ; 115 \leq \theta \leq 150$;

- sector 4: $50 \leq \phi \leq 90 ; 35 \leq \theta \leq 65$;

- sector 5: $50 \leq \phi \leq 90 ; 70 \leq \theta \leq 110$;

- sector 6: $50 \leq \phi \leq 90 ; 115 \leq \theta \leq 150$;

- sector 7: $0 \leq \phi \leq 90 ; 155 \leq \theta \leq 180$.

\section{A. Dataset Construction}

For each of the 5 sphere materials simulated in section III we create datasets that contain 8 classes corresponding to each sphere's angular sectors. In every dataset we include the 13 sphere sizes simulated in section III.

For the SF datasets in frequency and time domains, they are constructed as in section IV-A1 with same length. 

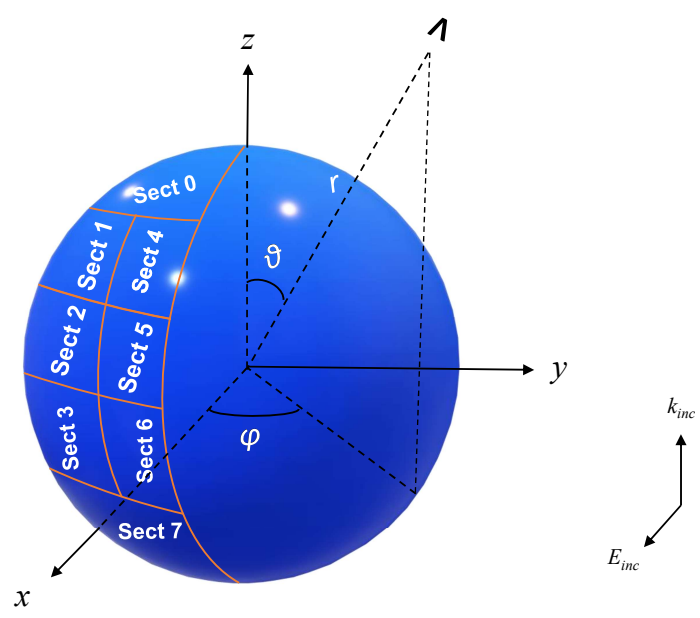

Fig. 9: Sphere's angular sectors

TABLE VIII: Parameters of SVM classifier

\begin{tabular}{cccc}
\hline \multirow{2}{*}{ Data } & \multicolumn{3}{c}{ Parameters } \\
& Function & $\mathrm{C}$ & $\gamma$ \\
\hline FD & rbf & 3500 & 100 \\
TD & rbf & 2500 & 100 \\
SEM & rbf & 1000 & 50 \\
\hline
\end{tabular}

For the SEM dataset, the input vector is also of length 5 and comprised of 2 channels representing the residues' amplitudes in $\theta$ and $\phi$ directions respectively. We eliminate the natural frequencies and the Q-factor as they do not vary with the aspect angle. Thus, each dataset holds 4810 samples $(37(\theta) \times 10(\phi) \times 13$ sphere sizes $)$.

The merits of using residues over raw SF data are: first, we have much less data in the input vector and second, the amplitude of the residues is independent of the object's size. Indeed, the natural frequencies at which the residues are derived are inversely proportional to the size of the sphere: For each sphere type, the residues are independent of its size since they are computed at the same electrical length. Consequently, they are unique to each sphere but informative about the angle aspect of the sphere. Thus, determining the observation angle using the residues will be faster than raw data, however, as there is less data, the residues might be more sensitive to noise.

\section{B. Classification results}

The results of the observation angle classification averaged over 10 runs are presented. As for the material classification process, we divide each dataset into $80 \%$ for train and $20 \%$ for test. Then we test the classification using noisy data and larger or smaller sphere sizes not included in the training datasets.

1) Training phase: As in section IV-B1, the SVM parameters are found by using 'gridsearch' function. Due to the complexity of the problem, a linear function does not achieve good results with the SEM dataset as before, hence we use the rbf kernel function.

The DT classifier's parameters are also left random. For the MLP classifier, we apply two hidden layers each with 64 and 256 neurons for SEM and SF data respectively. Increasing the layers number does not change the results. We apply the ReLU activation function in both layers and for all datasets (SF and
TABLE IX: Accuracy (\%) of observation angle for the $20 \%$ test sets

\begin{tabular}{ccccc}
\hline Data & SVM & DT & MLP & CNN \\
\hline FD & 97 & 52 & 96 & 97 \\
TD & 97 & 80 & 96 & 97 \\
SEM & 98 & 98 & 100 & 100 \\
\hline
\end{tabular}

SEM). For the CNN classifier, the LeNet-5 architecture is also adopted for the SF datasets, while for the SEM dataset we use one convolutional layer with 6 filters followed by one hidden layer with 64 neurons. The number of epochs is 256 for SEM data and 512 for SF data with a batch size of 64 for both MLP and CNN. The output layer is composed of 8 neurons using the softmax activation function.

2) Test phase: We start by testing the $20 \%$ remaining samples for the 5 datasets of each sphere. In fact, the results are almost the same for all spheres, hence, for brevity we present the results of the PEC sphere and compare the results of the SEM, FD and TD datasets. Table IX shows that SEM data has highest performances using ANN classifiers with $0 \%$ error rate. For SF data, there is 3\% error using the CNN and SVM, whereas DT has the lowest performances where Sens and Spec values do not exceed $60 \%$ for all classes, hence, DT is not a classifier that is suited for this problem. Fig. 10 shows the normalized confusion matrices using CNN. This first test, shows that identifying the position of the observer in regards to an angular sector of a sphere is possible when using the residues associated to each pole.

3) Noisy data: Then, we test the same noisy data simulated in section IV-B3. The CNN classifier has the highest performances for SF data, while for SEM data the CNN and MLP classifiers have similar performances. For 65 and $30 \mathrm{~dB}$ SNR, those classifiers applied to SEM data have a gain of $1 \%$ compared to those applied to SF data. Nevertheless, they have the lowest accuracy for 20 and $10 \mathrm{~dB}$ SNR where there is a loss of almost $12 \%$ compared to SF data (see fig. 11). In fact, as the SNR starts to decrease, the residues become highly perturbed as they are computed through the resonance poles which are also affected by noise. Only the residues associated to the first pole have the less distortions but as we will see in section V-B4, having one residue is not enough to determine the sphere's angular sector accurately. It is also observed that the miss-classified samples actually exist at the border of the sectors, thus it remains acceptable.

4) Generalization using different sphere sizes: Finally, we test the larger sphere sizes simulated in section IV-B. In fact, the residues' amplitude remains constant for different sizes as they are computed for the natural frequency of the SF response. On the other hand, the FD and TD responses depend upon the object's size. Fig. 12 shows that classifiers trained using both raw SF data are unable to determine the angular sectors of all spheres where the accuracy does not exceed $30 \%$ for the $30 \mathrm{~cm}$ diameter sphere. In addition, the Sens and Spec values of most classes do not exceed $40 \%$. On the contrary, classifiers trained using SEM data achieve high performances where the ANN classifiers have $0 \%$ and 3\% error for 19 and 30 $\mathrm{cm}$ diameters respectively. This confirms that classifiers trained 


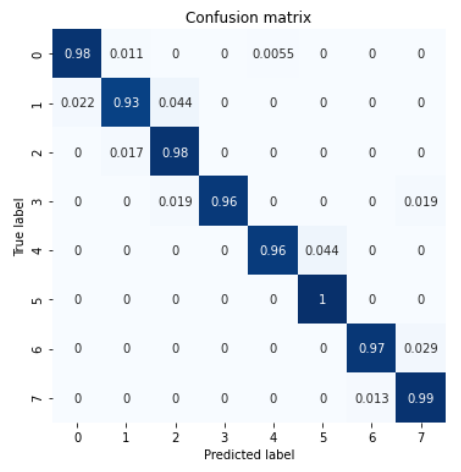

(a)

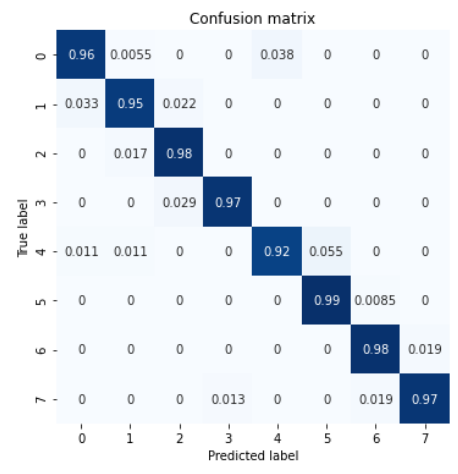

(b)

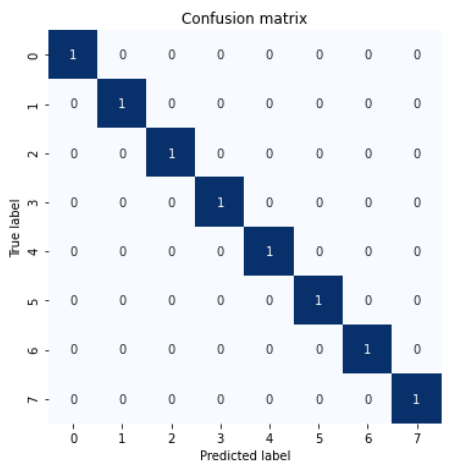

(c)

Fig. 10: Normalized confusion matrix using CNN classifier when classifying the observation angle of the PEC sphere using the $20 \%$ remaining samples of: (a) Frequency data; (b) Time data; (c) SEM data.

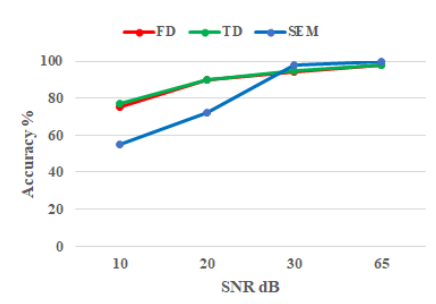

(a) SVM

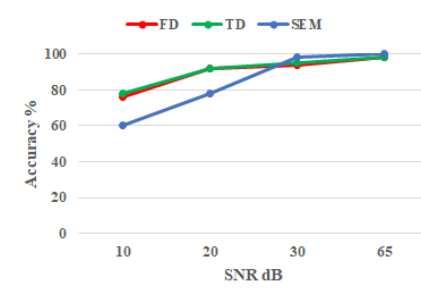

(c) MLP

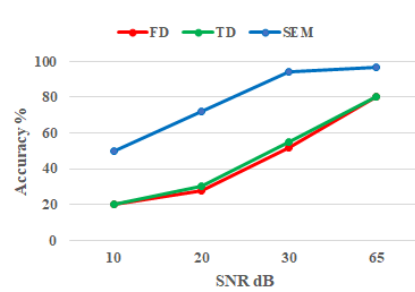

(b) DT

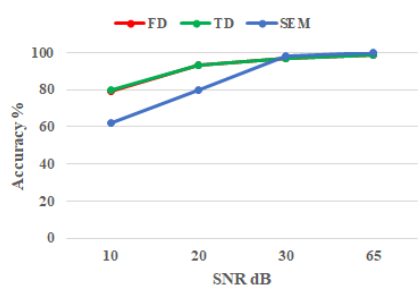

(d) $\mathrm{CNN}$
Fig. 11: Accuracy (\%) of noisy data when identifying the angular sectors of the PEC sphere

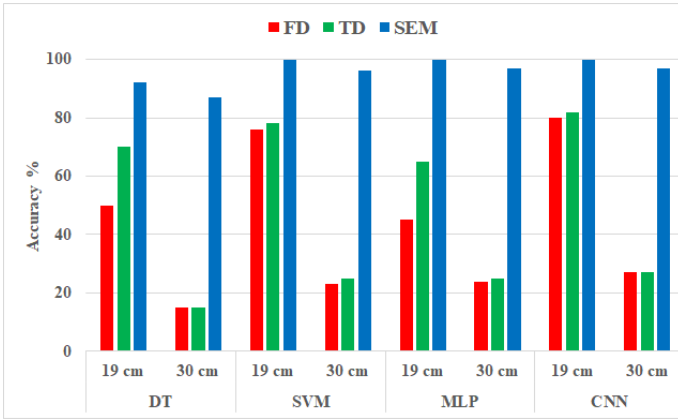

Fig. 12: Accuracy (\%) of angular sectors of larger PEC spheres

using SEM data are indeed able to identify the observation angle of larger spheres.

For smaller spheres, we test the classification capability on different sizes where the SEM test sets include $N<5$ natural frequencies (and thus less than 5 associated residues). The accuracy results are listed in table X. When the sphere's size decreases, classifiers trained using raw SF data become unable to detect the observation angle where for $\mathrm{N}=1$ (i.e. the smallest sphere) it is impossible to classify some classes as
TABLE X: Accuracy (\%) of observation angle for different number of resonances $\mathrm{N}$ of PEC sphere

\begin{tabular}{ccccc}
\hline \multirow{2}{*}{ Classifier } & \multirow{2}{*}{ N } & \multicolumn{3}{c}{ Data } \\
& & FD & TD & SEM \\
\hline \multirow{4}{*}{ CNN } & 4 & 95 & 95 & 99.7 \\
& 3 & 80 & 82 & 99.5 \\
& 2 & 33 & 48 & 94 \\
& 1 & 20 & 20 & 77 \\
\hline
\end{tabular}

TABLE XI: Accuracy (\%) of observation angle of different test data for 5 spheres using CNN classifier with SEM dataset

\begin{tabular}{cccccc}
\hline \multirow{2}{*}{ Test data } & PEC & $\epsilon_{r} \mathbf{= 4 ,} \sigma=\mathbf{0 . 5}$ & $\epsilon_{r}=\mathbf{2}$ & $\epsilon_{r}=\mathbf{4}$ & $\epsilon_{r}=\mathbf{9}$ \\
\hline$\varnothing \mathbf{3 0} \mathbf{~ c m}$ & 97 & 96 & 99 & 98 & 98 \\
$\mathbf{4}$ resonances & 99.7 & 99.5 & 99.2 & 98 & 98 \\
$\mathbf{3}$ resonances & 99.5 & 98.5 & 99.5 & 96 & 96 \\
$\mathbf{2}$ resonances & 94 & 90.8 & 90 & 90 & 90 \\
$\mathbf{1}$ resonance & 77 & 72 & 75 & 70 & 70 \\
$\mathbf{1 0}$ dB SNR & 62 & 57 & 58 & 55 & 56 \\
\hline
\end{tabular}

seen in fig.13a and 13b. We can note that the CNN classifier performs better than the rest, but it still has low accuracy when the sphere starts to have less than 4 resonances in the frequency band. For classifiers trained using SEM data, MLP and CNN classifiers perform best. It is observed that spheres with more than 1 resonance in the frequency band are easily classified with only $6 \%$ error for $\mathrm{N}=2$. However, when there is one resonance it is difficult to accurately determine some of the sphere's sectors through the residues associated to the first pole (fig. 13c).

Those results prove that using the residues to determine the position of the receiving antenna is actually possible even when there is much less information concerning the frequency spectrum compared to raw data. Even though we do not get performances better than the SF data when testing noisy data, this still remains a very promising result as we have 100 times and 20 times less data in the SEM dataset than the SF datasets in frequency and time domains respectively.

Table XI resumes the results obtained when classifying angular sectors of the 5 sphere types with ANN classifiers trained using noiseless SEM data. Those results are presented for the following test data: larger sphere size of $30 \mathrm{~cm}$ diameter $(\varnothing=30 \mathrm{~cm})$, smaller spheres having less than 5 resonances in the frequency band and noisy data of $10 \mathrm{~dB}$ SNR. 


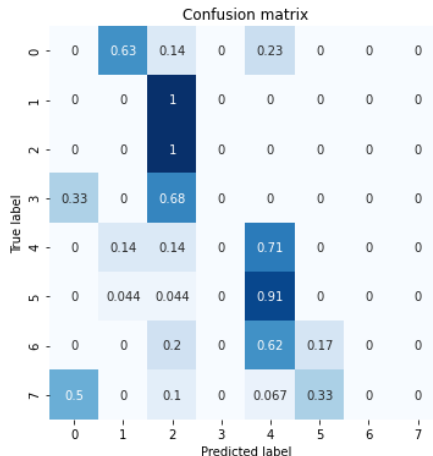

(a)

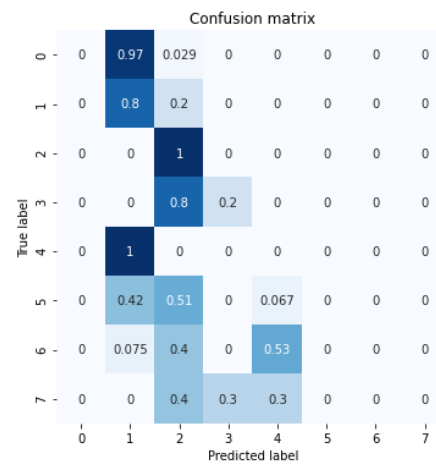

(b)

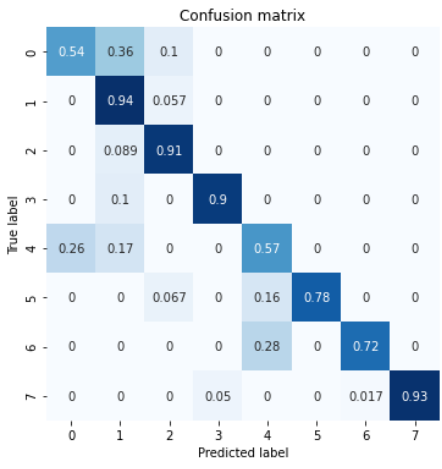

(c)

Fig. 13: Normalized confusion matrix using CNN classifier when classifying the observation angle of spheres having 1 resonance of: (a) Frequency data ; (b) Time data ; (c) SEM data.

\section{DISCUSSION}

Indeed, combining the SEM with a classification algorithm is an interesting research topic that has been seldom exploited and has not been applied to scattered fields emanating from all spatial directions. The work found in literature deals with simple classification cases: bi-class in [19] or multiclass in [20] but for the purpose of classifying four sizes of homothetic objects applied to limited directions of SF. These studies have highlighted not only the evident qualities of the SEM (excellent classification from few data and no matter the aspect angle of the target) but also its limitations to noise that affect the higher order poles. They have therefore limited their approach to the first one or two complex poles to build the dataset. Moreover, they have trained their classifiers at different SNR levels or have applied NRPCA, a noise reduction technique, as a pre-processing step, which is not very compatible with an operational context.

The approach in this paper is intended to be more global, with the objective of classifying the material of spherical objects of all sizes, from the scattered field in any direction, and without a priori knowledge about the nature of the noise. In this context, the structure of the proposed SEM input vector satisfies this objective. First, including the Q-factor and the residues associated to each natural pole improves the robustness and allows the generalization to different object sizes. Second, the sparsity in the SEM dataset (replacing higher order poles with zeros) is beneficial to overcome the bandwidth limitations for small objects and to improve the robustness to noise by decreasing the weight of higher order poles during the classification process. These performances, seen in section IV-B, were also achieved with the use of more advanced classification algorithms. Indeed, our study has shown that at SNR levels $\leq 20 \mathrm{~dB}$, the more basic algorithms (DT and SVM) do not take advantage of the additional information provided by the original format of the proposed SEM input vector where they miss-classify all samples of some classes. Conversely, ANNs are successful in taking advantage of the indirect but informative data (residues) and in handling sparse input data. The results presented indicate that combining ANN algorithms with VF can compensate for the weaknesses of SEM methods by surpassing the results obtained from FD or TD data even at low SNRs, without prior training with noisy data. In addition, the reduced SEM input vector size enables rapid convergence of the neural networks.

All these advantages open up new opportunities. First, by classifying the sphere's material, it is possible to determine the size of this sphere, which is directly related to the fundamental frequency of the first pole. Second, the classification of the observation angle from the residues is a novel problem that raises interesting perspectives, especially when applied to more complex objects than the sphere. This preliminary study, of identifying the observation angle, yielded significant advantages in terms of computational cost and generalizability despite the sensitivity of residues to noise. In fact, since the computation of the residues is independent of the size of the studied object, it is possible to construct the dataset from a single object size. This will reduce the processing time but more importantly, opens new prospects for the dataset construction as it is hard to compute the scattered fields in all directions for all dimensions of different objects.

However, some limitations can be noted in this study since, as in all papers dealing with SEM, we assume that the first natural frequency of the illuminated object is in the radar bandwidth, which implies a very low minimum frequency to classify large objects. Furthermore, the time required to compute the poles and residues by the VF algorithm may vary, mainly in low SNRs requiring more iterations and finer tuning of the initial parameters for a good convergence, which is to be integrated into the total computation time. Aware of these weaknesses, we believe that the gains in terms of processing time and computational cost remain largely in favour of the proposed approach. Moreover, considering the generalization to different object sizes and the improvements made in terms of robustness to noise, make this association a promising radar technique for object and observation angle classification.

\section{CONCLUSION}

The present work describes an efficient workflow to classify 5 different sphere materials and to determine the observation angle of the receiving antenna by dividing the sphere into 8 angular sectors. Three datasets are constructed based on the raw scattered field, in time and frequency domains, and the 
proposed pre-processed SEM data. They are constructed from multiple noiseless responses from several sphere sizes where the SEM data has a specific sparse input vector including the natural frequencies and their associated Q-factor and residues. The comparison between classification on SEM and raw datasets confirms that the proposed method allows to classify from a single observation angle while being efficient, aspect independent, and with low computational cost. Moreover, the use of Q-factor instead of damping factor allows to accurately distinguish spheres of different sizes not included in the training dataset. Finally, the sparsity of the SEM input vector associated with ANN classifiers allows also to maintain high classification rates even at low SNRs, without including noisy data in the training phase.

Following the sphere's material classification, we propose the use of residues to classify the observation angle of the identified object. The comparative classification results indicate that even though the SEM residues are affected by noise, the proposed approach offers excellent classification rates over FD or TD datasets whatever the size of the sphere. This generalization capability facilitates the dataset constitution and offers interesting perspectives in radar applications. It should also be noted that those performances could be greatly enhanced by including noisy data during the training phase. Hence, this preliminary study brought very promising results for future object classification with radar signal while using simpler but reliable and faster techniques than those using raw SF data. As a perspective, the study will be extended towards more complex non spherical objects. It will be also extended to an operational context by testing on measured scattered fields.

\section{REFERENCES}

[1] B. Schölkopf and A. Smola, Learning with Kernels: Support Vector Machines, Regularization, Optimization, and Beyond, MIT press, 2001.

[2] Chih-Wei Hsu and Chih-Jen Lin, "A comparison of methods for multiclass support vector machines," IEEE Transactions on Neural Networks, vol. 13, no. 2, pp. 415-425, 2002.

[3] W.-Y. Loh, "Classification and regression trees," Wiley Interdisciplinary Reviews: Data Mining and Knowledge Discovery, vol. 1, pp. 14-23, 01 2011.

[4] V. Sze, Y. Chen, T. Yang, and J. S. Emer, "Efficient processing of deep neural networks: A tutorial and survey," Proceedings of the IEEE, vol. 105, no. 12, pp. 2295-2329, 2017.

[5] E. Wengrowski, M. Purri, K. Dana, and A. Huston, "Deep cnns as a method to classify rotating objects based on monostatic rcs," Iet Radar Sonar and Navigation, vol. 13, pp. 1092-1100, 2019.

[6] T. D. Bufler, R. M. Narayanan, and T. Dogaru, "SVM based target classification using RCS feature vectors," vol. 9461. SPIE Proceedings, 2015, pp. $123-132$

[7] Yinan Yang, Jiajin Lei, Wenxue Zhang, and Chao Lu, "Target classification and pattern recognition using micro-doppler radar signatures," in Seventh ACIS International Conference on Software Engineering, Artificial Intelligence, Networking, and Parallel/Distributed Computing (SNPD'06), 2006, pp. 213-217.

[8] E. A. Hadhrami, M. A. Mufti, B. Taha, and N. Werghi, "Classification of ground moving radar targets using convolutional neural network," in 2018 22nd International Microwave and Radar Conference (MIKON), 2018, pp. 127-130.

[9] O. Chapelle, P. Haffner, and V. N. Vapnik, "Support vector machines for histogram-based image classification," IEEE Transactions on Neural Networks, vol. 10, no. 5, pp. 1055-1064, 1999.

[10] C. P. Schwegmann et al., "Subsidence feature discrimination using deep convolutional neural networks in synthetic aperture radar imagery," in 2017 IEEE IGARSS, 2017, pp. 4626-4629.
[11] C. E. Baum, E. J. Rothwell, K. . Chen, and D. P. Nyquist, "The singularity expansion method and its application to target identification," Proceedings of the IEEE, vol. 79, no. 10, pp. 1481-1492, 1991.

[12] J. Chauveau, N. de Beaucoudrey, and J. Saillard, "Selection of contributing natural poles for the characterization of perfectly conducting targets in resonance region," IEEE Transactions on Antennas and Propagation, vol. 55, no. 9, pp. 2610-2617, 2007.

[13] W. Lee, T. K. Sarkar, H. Moon, and M. Salazar-Palma, "Computation of the natural poles of an object in the frequency domain using the cauchy method," IEEE Antennas and Wireless Propagation Letters, vol. 11, pp. 1137-1140, 2012.

[14] Chi-Chih Chen, "Electromagnetic resonances of immersed dielectric spheres," IEEE Transactions on Antennas and Propagation, vol. 46, no. 7, pp. 1074-1083, 1998.

[15] J. Chauveau, N. de Beaucoudrey, and J. Saillard, "Modification of resonance poles of a conducting target by a dielectric coating," in 2007 European Radar Conference, 2007, pp. 440-443.

[16] W. Lee et al., "Effect of material parameters on the resonant frequencies of a dielectric object," IEEE Antennas and Wireless Propagation Letters, vol. 12, pp. 1311-1314, 2013.

[17] P. Leekul, M. Krairiksh, and T. K. Sarkar, "Application of the natural frequency estimation technique for mangosteen classification," in Asia-Pacific Conference on Antennas and Propagation, July 2014, pp. $928-930$.

[18] T. Tantisopharak et al., "Nondestructive determination of the maturity of the durian fruit in the frequency domain using the change in the natural frequency," IEEE Transactions on Antennas and Propagation, vol. 64, no. 5, pp. 1779-1787, 2016.

[19] Joon-Ho Lee, In-Sik Choi, and Hyo-Tae Kim, "Natural frequency-based neural network approach to radar target recognition," IEEE Transactions on Signal Processing, vol. 51, no. 12, pp. 3191-3197, 2003.

[20] J. A. Garzon-Guerrero, D. P. Ruiz, and M. C. Carrion, "Classification of geometrical targets using natural resonances and principal components analysis," IEEE Transactions on Antennas and Propagation, vol. 61, no. 9, pp. 4881-4884, 2013.

[21] F. Pedregosa et al., "Scikit-learn: Machine learning in python," Journal of Machine Learning Research, vol. 12, 012012.

[22] G. Mie, "Beiträge zur optik trüber medien, speziell kolloidaler metallösungen," Annalen der Physik, vol. 330, pp. 377-445, 1908.

[23] B. Gustavsen and A. Semlyen, "Rational approximation of frequency domain responses by vector fitting," IEEE Transactions on Power Delivery, vol. 14, no. 3, pp. 1052-1061, July 1999.

[24] Y. Y. Zaky et al., "Comparison of sem methods for poles estimation from scattered field by canonical objects," in IEEE Radar Conference (RadarConf20), 2020, pp. 1-6.

[25] D. Deschrijver, M. Mrozowski, T. Dhaene, and D. De Zutter, "Macromodeling of multiport systems using a fast implementation of the vector fitting method," IEEE Microwave and Wireless Components Letters, vol. 18, no. 6, pp. 383-385, June 2008.

[26] B. Gustavsen, "Improving the pole relocating properties of vector fitting," IEEE Trans. on Power Delivery, vol. 21, no. 3, pp. 1587-1592, July 2006.

[27] J. Chauveau, N. de Beaucoudrey, and J. Saillard, "Study of resonances of scattering objects: comparison with resonant circuits and characterization using q-factor," in IEEE AP-S International Symposium, 2006, pp. 3271-3274.

[28] D. Kingma and J. Ba, "Adam: A method for stochastic optimization," International Conference on Learning Representations, 122014.

[29] Y. Lecun, L. Bottou, Y. Bengio, and P. Haffner, "Gradient-based learning applied to document recognition," Proceedings of the IEEE, vol. 86, no. 11 , pp. $2278-2324,1998$. 